\title{
Mastectomía ahorradora de piel y pezón en carcinoma ductal in situ. Seguridad oncológica a 10 años
}

\section{Marta Allué Cabañuz ${ }^{\mathrm{a}, *}$, Jorge Chóliz Ezquerro ${ }^{\mathrm{b}}$, Maria Dolores Arribas del Amo ${ }^{\mathrm{a}}$, Ismael Gil Romea ${ }^{a}$, María Pilar Val-Carreres Rivera ${ }^{a}$, Ramón Sousa Domínguez ${ }^{a}$ y Antonio Tomás Guiemes Sánchez ${ }^{a}$}

\author{
a Unidad de Mama, Hospital Clínico Universitario Lozano Blesa, Zaragoza, España \\ ${ }^{\mathrm{b}}$ Hospital Universitario Miguel Servet, Zaragoza, España
}

Recibido el 20 de marzo de 2020; aceptado el 3 de abril de 2020

\section{PALABRAS CLAVE \\ Carcinoma ductal \\ in situ; \\ Mastectomía con \\ preservación del \\ pezón; \\ Reconstrucción mamaria inmediata; \\ Cirugía mamaria; \\ Recidiva}

\begin{abstract}
Resumen
Objetivo: El objetivo es evaluar la seguridad oncológica a 10 años de la mastectomía ahorradora de piel y pezón (MAP) en pacientes con carcinoma ductal in situ (CDIS).

Método: Análisis observacional retrospectivo. Se realizaron 35 MAP en pacientes con CDIS durante 2005-2018. Evaluamos resultados histológicos, oncológicos y de morbilidad.

Resultados: Las indicaciones más frecuentes fueron márgenes afectos tras tumorectomía $(31,5 \%)$, multifocalidad/multicéntricidad $(22,8 \%)$, tumor $>3 \mathrm{~cm}(8,6 \%)$ correlación desfavorable tamaño tumoral/mama $(8,6 \%)$ y decisión de la paciente $(8,6 \%)$. La técnica más usada fue incisión lateral externa en 11 pacientes, seguida de técnica de Spira en nueve casos.

La presencia de CDIS se confirmó en 22 pacientes y en 11 no se encontró tumor en la pieza de mastectomía.

La tasa de complicaciones fue $22,8 \%$. Tras una mediana de seguimiento de 104 meses (DE $69,9)$ no se observó necrosis del pezón. Un $20 \%$ de pacientes precisó reintervención a largo plazo. Once pacientes $(31,4 \%)$ recibieron tratamiento adyuvante (QT y/o RT). Solamente una paciente presentó recurrencia local (2,8\%) 28 meses tras la intervención. Una paciente presentó metástasis tras 78 meses de SLE. Las tasas de SLE y SG fueron $94,3 \%$ y $97,22 \%$.

El análisis univariante mostró dos factores de riesgo de recurrencia: edad <40 [OR (IC95) $2,529(1,230-5,199)$ ] y márgenes afectos [OR (IC95) 5,242 $(2,041-13,464)$ ].

Conclusión: La MAP es factible y segura en pacientes con CDIS no candidatas a cirugía conservadora.

(c) 2020 SESPM. Publicado por Elsevier España, S.L.U. Todos los derechos reservados.
\end{abstract}

\footnotetext{
* Autor para correspondencia.

Correo electrónico: martitaallue@hotmail.com (M. Allué Cabañuz).
} 


\section{KEYWORDS}

Ductal carcinoma

in situ;

Nipple-sparing

mastectomy;

Immediate breast

reconstruction;

Breast surgery;

Recurrence
Nipple-sparing mastectomy in ductal carcinoma in situ. Oncological results over a 10-year period

\footnotetext{
Abstract

Objective: The aim of this study was to assess the oncological safety of nipple-sparing mastectomy (NSM) in patients with ductal in situ carcinoma (DCIS) over a 10-year period.

Method: Retrospective observational analysis. A total of 35 NSM were performed in patients with DCIS from 2005 - 2018. We assessed the histological, oncological and morbidity results.

Results: The most frequent indications were margin involvement after lumpectomy (31.5\%), multifocality / multicentricity (22.8\%), tumour size $>3 \mathrm{~cm}(8.6 \%)$ unfavourable tumour / breast size correlation $(8.6 \%)$ and patient choice $(8.6 \%)$. The most commonly used technique was external lateral incision in 11 patients, followed by the Spira technique in 9 patients.

DCIS was confirmed in 22 patients and no tumour was found in mastectomy specimen in 11 patients.

The complication rate was $22.8 \%$. After a median follow-up of 104 months (SD 69.9) there was no nipple necrosis. In all, $20 \%$ of the patients required long-term reintervention. Eleven patients $(31.4 \%)$ underwent adjuvant treatment (chemotherapy and / or radiotherapy). Only one patient showed local recurrence $(2.8 \%) 28$ months after the intervention. One patient developed metastases after 78 months of disease-free survival (DFS). DFS and overall survival rates were $94.3 \%$ and $97.22 \%$.

Univariate analysis showed two risk factors for recurrence: age <40 years [OR (95\% CI) 2.529 (1.230-5.199)] and margin involvement [OR (95\% CI) $5.242(2.041-13.464)]$.

Conclusion: NSM is safe and feasible in patients with DCIS who are not candidates for conservative surgery.

(c) 2020 SESPM. Published by Elsevier España, S.L.U. All rights reserved.
}

\section{Introducción}

La cirugía conservadora seguida de radioterapia constituye el gold standard para el tratamiento quirúrgico del cáncer de mama localizado y en estadios precoces. A pesar de los avances conseguidos en cirugía conservadora mediante la generalización de las técnicas de oncoplastia, hasta un 25\% de mujeres son sometidas a mastectomía ${ }^{1}$, debido a tumores grandes, multicéntricos, a una correlación entre el tamaño del tumor y la mama que impide la conservación de la misma o en pacientes que presentan recurrencia tras cirugía conservadora previa.

Mientras la mastectomía ahorradora de piel (MAP) está ampliamente validada para el tratamiento del cáncer de mama $^{2,3}$, la preservación del complejo aréola pezón (CAP) sigue generando controversia ${ }^{4}$. Los resultados oncológicos en términos de recurrencia y las tasas de complicaciones, fundamentalmente la necrosis del pezón, siguen generando preocupación a pesar de los beneficios cosméticos y en calidad de vida para las pacientes (reduciendo la necesidad de reconstrucción, tatuajes, etc.) que aporta la preservación del CAP ${ }^{5}$.

El CDIS, a pesar de tratarse de un tumor no invasivo, se caracteriza por su localización difusa, pudiendo presentar tejido neoplásico próximo al CAP, con el consiguiente riesgo de recurrencia a largo plazo.

El objetivo del estudio fue determinar la seguridad oncológica a 10 años de la MAP en pacientes con CDIS y analizar nuestra experiencia durante los últimos años, con énfasis en indicaciones, complicaciones y tasas de recidiva.

\section{Material y método}

Análisis observacional retrospectivo. Pacientes con CDIS intervenidas entre 2005 - 2018.

Se recopilaron datos sobre variables demográficas (edad, comorbilidades como obesidad, HTA [hipertensión arterial], DM [diabetes mellitus] y tabaquismo activo), clínicopatológicas (indicación, estadio clínico, etc.), en relación al tratamiento quirúrgico incluyendo el tipo de intervención, técnicas reconstructivas empleadas y la morbilidad postoperatoria, así como resultados oncológicos.

Este estudio se adhiere a los principios éticos de la Declaración de Helsinki y fue aprobado por el comité ético del Centro y por el Comité Ético de Investigación Clínica de Aragón (CEICA) con número de registro C.P. - C.I. PI16/002.

\section{Selección de pacientes}

Los criterios de inclusión fueron los siguientes:

- CDIS mayor de $3 \mathrm{~cm}$.

- Correlación desfavorable entre tamaño tumoral y tamaño de la mama que impida realizar cirugía conservadora con adecuado resultado cosmético.

- Tumor multifocal (dos o más focos tumorales en un mismo cuadrante y a menos de 5 centímetros del foco primario) o multicéntrico (dos o más focos tumorales en distintos cuadrantes de la misma mama o a más de 5 centímetros del foco primario) no susceptible de cirugía conservadora. 
- Recurrencia tras tratamiento previo por CDIS (mediante cirugía conservadora y RT).

- Alto riesgo por historia familiar (sin mutación conocida). Definido por dos o más familiares (al menos uno de ellos de primer grado) afectados de cáncer de mama u ovario a edades tempranas, antes de los 50 años.

- Mutación conocida en genes BRCA 1 y 2.

- Tumores a más de $2 \mathrm{~cm}$ de distancia del CAP, determinado mediante imagen preoperatoria (mamografía y/o RMN [resonancia magnética nuclear]).

Se excluyeron pacientes con carcinoma inflamatorio, enfermedad de Paget del pezón, infiltración clínica del pezón o secreción patológica del pezón y metástasis a distancia al diagnóstico.

\section{Técnica quirúrgica}

La técnica empleada fue similar en todas las pacientes realizando resección de tejido glandular mamario, preservando finos colgajos de piel y evitando la utilización de electrocoagulación en áreas próximas a la base del pezón (sustituyéndolo por el uso de tijera y bisturí frío) y respetando un espesor mínimo de $1 \mathrm{~cm}$. Para corroborar la ausencia de afectación de este tejido se realiza biopsia intraoperatoria, obteniendo el material mediante eversión del pezón o sección en los casos de posterior injerto libre del CAP. En caso de afectación se desestima la MAP con preservación del CAP. Asimismo se evaluó la viabilidad del tejido areolar mediante examen visual $y$, en caso de perfusión inadecuada, no se preservó el CAP.

Las incisiones empleadas varían en función del tamaño y grado de ptosis de la mama afecta y la contralateral, existencia de cicatrices previas y preferencias del cirujano (fig. 1).

- Mastectomía subcutánea por incisión lateral externa.

- Técnica de Spira modificada: prótesis con doble cubierta mediante un colgajo desepitelizado unido al músculo pectoral mayor e injerto libre del CAP tras biopsia intraoperatoria negativa de la base del pezón.

- Mastectomía ahorradora de piel según patrón de Wise corto: Incisión periareolar con prolongación vertical hacia el surco submamario y extensión lateral y medial a lo largo del surco.

- Mastectomía ahorradora de piel y pezón por incisión radial externa.

La reconstrucción inmediata es llevada a cabo mediante la colocación de implante de silicona de perfil anatómico alojado en posición retromuscular, o en un bolsillo creado suturando el músculo pectoral ( $2 / 3$ superiores) a un colgajo dermograso que cubre el tercio inferior de la prótesis en la técnica de Spira (fig. 2).

La necrosis del CAP se definió como parcial si consiguió recuperarse, y total si requirió extirpación quirúrgica con reconstrucción secundaria.

\section{Evaluación patológica y resultados oncológicos}

La administración de quimioterapia (QT) o radioterapia (RT) adyuvante se ajustó a las guías de práctica clínica del Centro en el momento del tratamiento.

La recurrencia locorregional se definió como aparición de nuevo tumor en la pared torácica ipsilateral (piel, tejido subcutáneo y músculo pectoral) o recurrencia en la axila ipsilateral, ganglios linfáticos supraclaviculares, en cadenas mamarias internas o infraclaviculares.

La recidiva a distancia (metástasis) fue definida como cualquier recurrencia en todas las demás áreas no incluidas en la recurrencia locorregional.

\section{Análisis estadístico}

En primer lugar, se realiza un análisis descriptivo de las variables a estudio. Para variables cualitativas se aportan frecuencias relativas y absolutas. Para variables cuantitativas, media y desviación estándar (DE).

Para evaluar la supervivencia global (SG), supervivencia libre de enfermedad (SLE), tasa de recidiva se utilizó el test de Log-Rank. Se calculó la SLE como el tiempo desde la intervención quirúrgica hasta la aparición de recaída local o sistémica durante el periodo de estudio. Se presentó un tipo de censura (C) que fue la falta de ocurrencia del evento al finalizar el periodo de observación.

Se establece el nivel de significación estadística para un valor de $p$ menor a 0,05. Para todo el análisis se ha utilizado el programa estadístico SPSS 22.0 para Windows (SPSS Ibérica, Madrid, España).

\section{Resultados}

\section{Resultados generales}

Se intervinieron un total de 35 pacientes con CDIS mediante MAP bilateral en el periodo de estudio. La mediana de seguimiento fue de 104 meses (DE 69,9). La edad media de las pacientes fue de 49,7 $\pm 8,7$ años. Fueron estudiadas las comorbilidades que pudieran afectar al desarrollo de complicaciones: siete pacientes (20\%) eran hipertensas, dos $(5,7 \%)$ diabéticas, cinco $(14,3 \%)$ fumadoras y dos $(5,7 \%)$ obesas, con importante grado de ptosis mamaria (tabla 1 ).

Las indicaciones más frecuentes fueron márgenes afectos tras tumorectomía (31,5\%), multifocali$\mathrm{dad} /$ multicentricidad $(22,8 \%)$, tumor $>3 \mathrm{~cm}(8,6 \%)$ correlación desfavorable tamaño tumoral/mama $(8,6 \%)$ y decisión de la paciente $(8,6 \%)$. Trece pacientes $(37,1 \%)$ habían sido tratadas por CDIS previo mediante cirugía conservadora, 11 de ellas presentaron márgenes próximos o afectos y optaron por la mastectomía en lugar de una nueva escisión, y dos pacientes presentaban recurrencia tras cirugía conservadora + RT previa (11 y 13 meses antes). El resto se detallan en la tabla 1.

\section{Técnica quirúrgica y complicaciones}

La técnica más usada fue MAP mediante incisión lateral externa en 11 pacientes, seguida de técnica de Spira modi- 


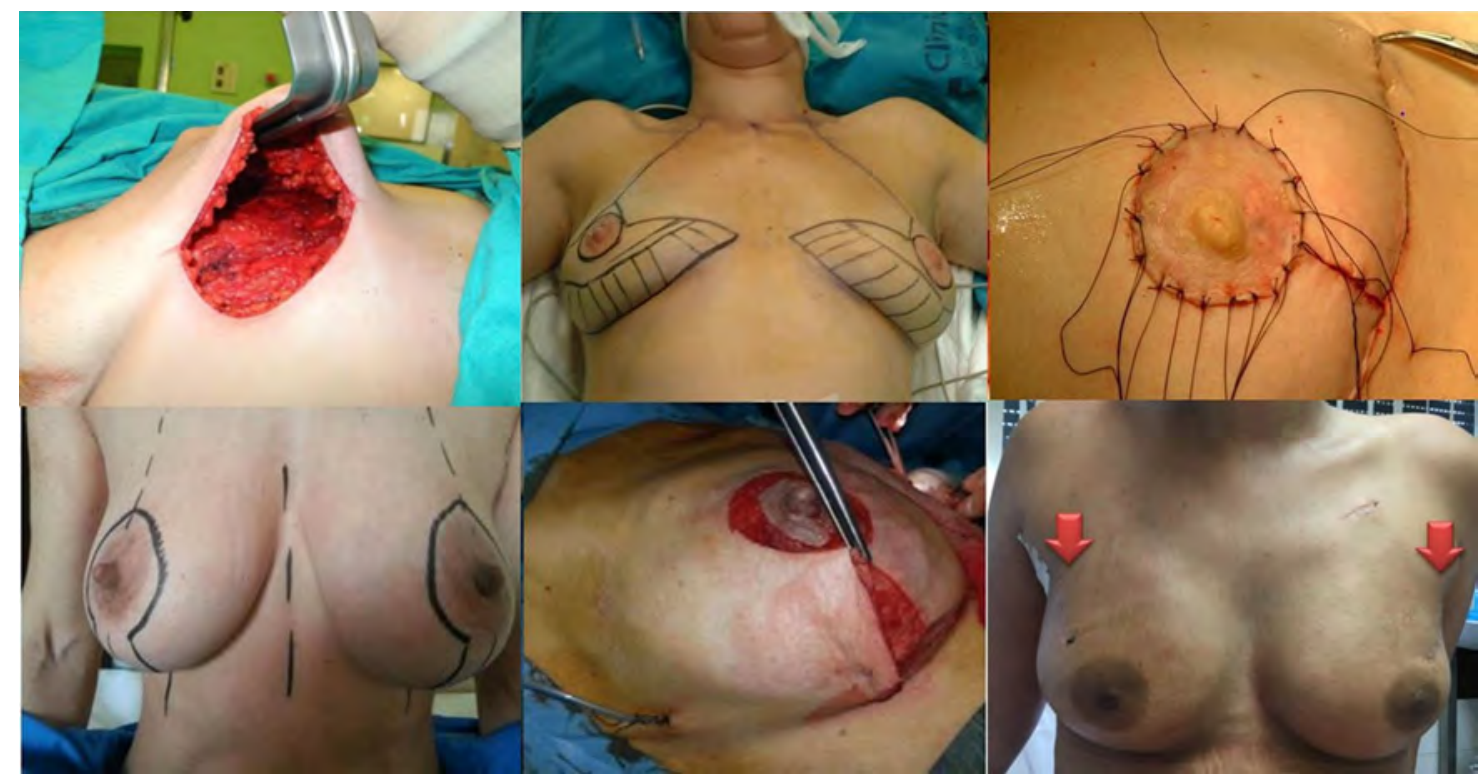

Figura 1 Diferentes técnicas de MAP empleadas. a) Mastectomía subcutánea por incisión lateral externa; b) técnica de Spira modificada; c) injerto libre del CAP en técnica de Spira; d) Patrón de Wise corto; e) cierre patrón cutáneo de Wise, y f) incisión radial externa.

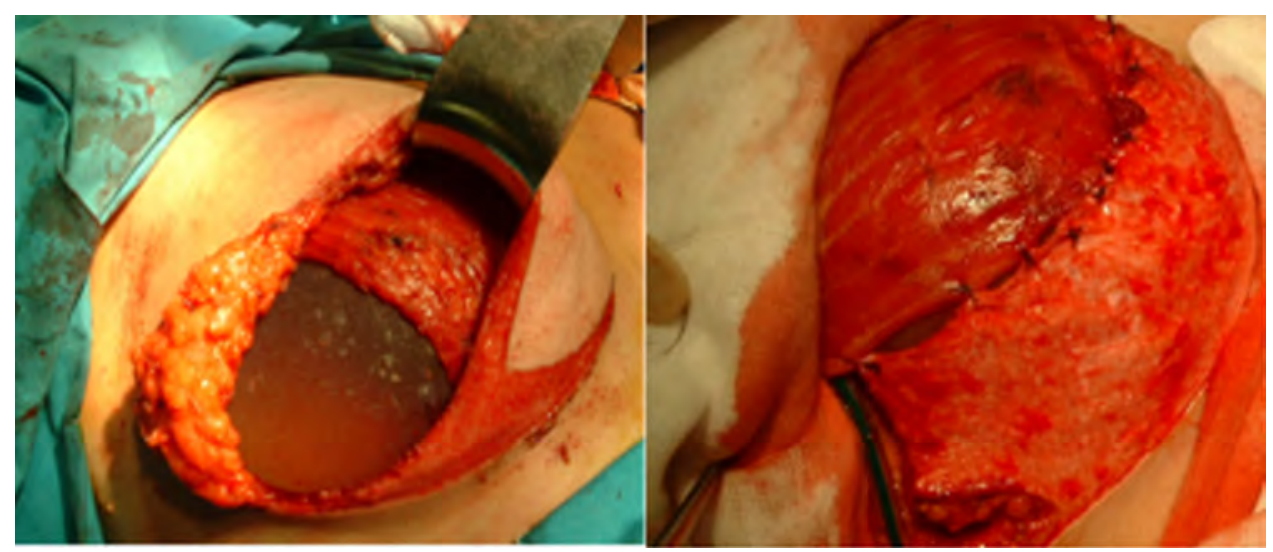

Figura 2 Reconstrucción mediante implante directo alojado en bolsillo retropectoral y cubierto inferiormente por colgajo dermograso.

ficada con injerto libre del CAP en nueve casos, patrón de Wise corto en cinco e incisión radial externa en dos pacientes (tabla 2).

La tasa de complicaciones fue del $22,8 \%$, siendo las más frecuentes necrosis cutánea y hematoma $(8,6 \%)$. No se observó ningún caso de necrosis del pezón. Un $20 \%$ de pacientes precisó reintervención a largo plazo por mal resultado cosmético asociado a secuelas (contractura capsular periprotésica y asimetría). Los resultados de comorbilidad se exponen en la tabla 2.

\section{Hallazgos anatomopatológicos en piezas de mastectomía}

La presencia de CDIS se confirmó en 22 pacientes, en dos se encontró carcinoma ductal infiltrante (CDI); ptTN1 y pT2n1, y en 11 no se encontró tumor en la pieza de mastectomía, probablemente fue extirpado mediante biopsia escisional previa. El tamaño tumoral medio fue de $1,33 \mathrm{~cm}$ (rango 0,2$3,5 \mathrm{~cm}$ ). Se realizó biopsia selectiva del ganglio centinela en 13 casos, siendo únicamente positivo en dos pacientes, que corresponden con los casos de CDI, en las que se completó la linfadenectomía axilar (tabla 3).

\section{Resultados oncológicos}

Un total de 11 pacientes $(31,4 \%)$ recibieron tratamiento adyuvante (QT y/o RT). Solamente una paciente presentó recurrencia local $(2,8 \%) 28$ meses tras la intervención. Una paciente presentó metástasis tras 78 meses de SLE. Las tasas de SLE y SG fueron $94,3 \%$ y $97,22 \%$, respectivamente (tabla 3).

El análisis univariante mostró dos factores de riesgo de recurrencia: edad <40 [OR (IC95) 2,529 (1,230 - 5,199)] 
Tabla 1 Datos demográficos, comorbilidades e indicaciones de la MAP en CDIS

\begin{tabular}{ll}
\hline Datos generales & $\mathrm{n}=35$ \\
\hline Edad & $49,7 \pm 8,7$ años \\
HTA & $7(20 \%)$ \\
DM & $2(5,7 \%)$ \\
Tabaquismo & $5(14,3 \%)$ \\
Obesidad & $2(5,7 \%)$ \\
Historia previa de cáncer de mama & $13(37,1 \%)$ \\
Cirugía conservadora por cáncer de mama & $13(37,1 \%)$ \\
previa & \\
Quimioterapia adyuvante & $7(20 \%)$ \\
Radioterapia adyuvante & $4(11,4 \%)$ \\
& \\
Indicaciones & $\mathrm{n}=35$ \\
\hline Tumor $>3$ cm & $3(8,6 \%)$ \\
Correlación desfavorable tamaño & $3(8,6 \%)$ \\
tumor/mama & \\
Tumor MF y/o MC & $8(22,8 \%)$ \\
Recurrencia tras CC previa & $2(5,7 \%)$ \\
Márgenes afectos tras CC previa & $11(31,5 \%)$ \\
Mutación BRCA1/2 & $2(5,7 \%)$ \\
Alto riesgo sin mutación & $3(8,6 \%)$ \\
Elección de la paciente & $3(8,6 \%)$ \\
\hline
\end{tabular}

HTA: hipertensión arterial; DM: diabetes mellitus; MF: multifocalidad;

MC: multicentralidad; CC: cirugía conservadora.

Tabla 2 Técnica quirúrgica utilizada y resultados

\begin{tabular}{ll}
\hline Técnica quirúrgica y resultados & $\mathrm{n}=35$ \\
\hline SPIRA modificado & $16(45,7 \%)$ \\
Incisión lateral externa & $12(34,3 \%)$ \\
Wise corto & $5(14,3 \%)$ \\
Incisión radial externa & $2(5,7 \%)$ \\
Complicaciones & $\mathrm{n}=8 / 35(22,8 \%)$ \\
\hline Necrosis cutánea & $3(8,6 \%)$ \\
Seroma & $2(5,7 \%)$ \\
Hematoma & $3(8,6 \%)$ \\
Secuelas & $\mathrm{n}=7 / 35(20 \%)$ \\
\hline Contractura capsular & $5(14,3 \%)$ \\
Asimetría & $2(5,7 \%)$ \\
Reintervención a largo plazo & $7(20 \%)$ \\
\hline
\end{tabular}

y márgenes afectos [OR (IC95) 5,242 (2,041 - 13,464)] (tabla 4).

\section{Discusión}

A pesar de la resección de casi la totalidad del tejido glandular mamario con las diferentes técnicas de mastectomía preservadora de piel y pezón, todavía se discute su seguridad oncológica por la posible participación del CAP. La teoría se basa en el hecho de que existen unidades lobulares ductales terminales (TDLU) en el tejido del CAP, hasta en un 25\% de los especímenes analizados ${ }^{6}$.
Tabla 3 Hallazgos anatomopatológicos y resultados oncológicos

\begin{tabular}{ll}
\hline Hallazgos anatomopatológicos & $\mathrm{n}=35$ \\
\hline No tumor & $11(31,4 \%)$ \\
CDIS & $22(62,8 \%)$ \\
Invasivo (CDI) & $2(5,7 \%)$ \\
$\begin{array}{l}\text { Tamaño tumoral cm, media } \\
\quad \text { rango) }\end{array}$ & $1,33(0,2-3,5)$ \\
Estado ganglionar & \\
$\quad$ No realizado ganglio & $22(62,8 \%)$ \\
$\quad$ centinela & \\
$\quad$ Negativo & $11(31,4 \%)$ \\
$\quad$ Positivo & $2(5,7 \%)$ \\
Resultados oncológicos & $\mathrm{n}=35$ \\
\hline Recidiva local & $1(2,8 \%)$ \\
Recidiva a distancia & $1(2,8 \%)$ \\
Recidiva pezón & 0 \\
SLE media & 92 meses (rango 28-155) \\
SLE & $94,3 \%$ \\
SG & 121 meses (rango 78-155) \\
SG & $97,2 \%$ \\
\hline
\end{tabular}

CDIS: carcinoma ductal in situ; CDI: carcinoma ductal infiltrante; SLE: supervivencia libre de enfermedad; SG: supervivencia general.

Tabla 4 Modelos de regresión univariante. Se muestran únicamente resultados con significación estadística

\begin{tabular}{ll}
\hline Factores predictores de recurrencia & OR (IC 95\%) \\
\hline Edad al diagnóstico < 40 años & $2,529(1,230-5,199)$ \\
Multicentricidad & \\
Multifocalidad & \\
Riesgo familiar & \\
BRCA $1 / 2$ & \\
RT / QT adyuvante & $5,242(2,041-13,464)$ \\
Márgenes afectos o < $1 \mathrm{~mm}$ & \\
Tumor > $3 \mathrm{~cm}$ & \\
\hline RT: radioterapia; QT: quimioterapia.
\end{tabular}

La biopsia intraoperatoria del tejido de la base del CAP es una herramienta muy útil para descartar la presencia de patología retroareolar, dada su alta sensibilidad y especificidad y su baja tasa de falsos negativos. Además, en caso de producirse un resultado falso negativo, la tasa de recurrencia locorregional en estas pacientes es baja ${ }^{7}$.

En nuestra serie de 35 pacientes, a las que realizamos biopsia intraoperatoria, encontramos sólo un caso de recurrencia durante un periodo de seguimiento medio de 8,6 años.

En la literatura publicada, la mayoría de trabajos cuentan con muestras heterogéneas (carcinoma in situ, cáncer invasivo, mastectomías profilácticas). Leclère et al. ${ }^{8}$ publican un estudio de 41 pacientes con CDIS sometidas a MAP, de las cuales sólo en 19 casos se pudo preservar el pezón (en el resto se descartó por localizarse el tumor próximo al CAP) describiendo una tasa de recurrencia local del 5,3\% con un seguimiento medio de 7,1 años. 
Teóricamente, el CDIS no tiene la capacidad de desarrollar metástasis, pero podemos encontrar CDIS en ganglios axilares por la teoría del transporte mecánico de las células epiteliales, aunque la realización de biopsia selectiva de ganglio centinela de rutina en estas pacientes no está indicado, salvo en casos de alto riesgo de enfermedad invasiva concomitante $^{9,10}$. Nuestro estudio comprende un periodo largo de tiempo en el que los estándares con respecto al tratamiento de la axila en el CDIS han cambiado.

Estamos asistiendo a un constante progreso en investigación genética y genómica, tratando de identificar pacientes con alto riesgo de desarrollar recurrencia local ${ }^{11}$ pero todavía no existen datos concluyentes que permitan extrapolar a la práctica clínica. Actualmente, no existen estudios controlados aleatorios que comparen la MAP, con o sin preservación del CAP y la mastectomía convencional en CDIS, ya que resultarían éticamente cuestionables. Sólo estudios con seguimiento a largo plazo podrán definir la seguridad oncológica de esta técnica en estas pacientes.

Por otra parte, muchos autores cuestionan la preservación del CAP en presencia de factores de riesgo tales como tabaquismo, ptosis mamaria (en pacientes obesas) e incisión periareolar ${ }^{12}$. La incidencia reportada de necrosis del CAP en MAP y pezón varía de $6,3 \%$ a $17,1 \%{ }^{13}$. En el estudio antes citado sobre $\mathrm{CDIS}^{8}$, la tasa de necrosis del CAP (con pérdida del mismo) era del $17 \%$. En nuestra serie encontramos necrosis cutáneas que se resolvieron de forma conservadora y ningún caso de necrosis del CAP, inclusive en pacientes con estos factores de riesgo.

Al igual que en el estudio publicado por Lago et al. ${ }^{14}$ con una baja tasa de recidiva del pezón $(1,4 \%)$ y una buena tasa de SG $(98,6 \%)$ tras un periodo de seguimiento medio de 142,6 meses, nuestras pacientes alcanzaron una tasa de SG del $97,22 \%$ tras una mediana de seguimiento de 104 meses (DE $69,9)$.

Este estudio cuenta con limitaciones al tratarse de un análisis retrospectivo, sin grupo control. Actualmente no existen estudios controlados aleatorios que comparen la MAP con preservación del CAP con la mastectomía convencional para CDIS pues resultaría éticamente cuestionable, por lo que este trabajo aporta información útil al contar con un periodo de seguimiento amplio.

\section{Conclusiones}

No se observó ningún caso de necrosis ni de recidiva del pezón. Se alcanzó una buena tasa de supervivencia global $(97,22 \%)$ tras una mediana de seguimiento de 104 meses (DE $69,9)$. La MAP es factible y segura en pacientes con CDIS no candidatas a cirugía conservadora.

\section{Conflicto de intereses}

Los autores declaran no tener ningún conflicto de intereses.

\section{Agradecimientos}

A los integrantes de Unidad de Mama del Hospital Clínico Universitario Lozano Blesa de Zaragoza, a cirujanos, personal de enfermería y a las pacientes.

\section{Bibliografía}

1. Mallon P, Feron JG, Couturaud B, Fitoussi A, Lemasurier P, Guihard T, et al. The role of nipple-sparing mastectomy in breast cancer: a comprehensive review of the literature. Plast Reconstr Surg. 2013;131:969-84.

2. Gerber B, Krause A, Reimer T, Müller H, Küchenmeister I, Makovitzky J, et al. Skin-sparing mastectomy with conservation of the nipple-areola complex and autologous reconstruction is an oncologically safe procedure. Ann Surg. 2003;238:120-7.

3. Garwood E, Moore D, Ewing C, Hwang ES, Alvarado M, Foster RD, et al. Total skin-sparing mastectomy: complications and local recurrence rates in 2 cohorts of patients. Ann Surg. 2009;249:26-32.

4. Ziogas D, Roukos DH, Zografos GC. Nipple-sparing mastectomy: overcoming oncological outcomes challenges. Ann Surg Oncol. 2010;17:323-4.

5. Wellisch DK, Schain WS, Noone RB, Little JW $3^{\text {rd }}$. The psychological contribution of nipple addition in breast reconstruction. Plast Reconstr Surg. 1987;80:699-704.

6. Kryvenko ON, Yoon JY, Chitale DA, Lee MW. Prevalence of terminal duct lobular units and frequency of neoplastic involvement of the nipple in mastectomy. Arch Pathol Lab Med. 2013;137:955-60.

7. Kneubil MC, Lohsiriwat V, Curigliano G, Brollo J, Botteri E, Rotmensz $\mathrm{N}$, et al. Risk of locoregional recurrence in patients with false-negative frozen section or close margins of retroareolar specimen in nipple-sparing mastectomy. Ann Surg Oncol. 2012;19:4117-23.

8. Leclère FM, Panet-Spallina J, Kolb F, Garbay JR, Mazouni C, Leduey A, et al. Nipple-sparing mastectomy and immediate reconstruction in ductal carcinoma in situ: a critical assessment with 41 patients. Aesthetic Plast Surg. 2014;38:338-43.

9. Francis AM, Haugen CE, Grimes LM, Crow JR, Yi M, Mittendorf EA, et al. Is sentinel lymph node dissection warranted for patients with a diagnosis of ductal carcinoma in situ? Ann Surg Oncol. 2015;22:4270-9.

10. Rubio I, Roca I, Sabadell D, Xercavins J. Beneficio de la biopsia del ganglio linfático centinela en pacientes con carcinoma in situ de mama. Cir Esp. 2009;85:92-5.

11. Roukos DH. Breast cancer outcomes: the crucial role of the breast surgeon in the era of personal genetics and systems biology. Ann Surg. 2009;249:1067-8.

12. Algaithy ZK, Petit JY, Lohsiriwat V, Maisonneuve P, Rey PC, Baros $\mathrm{N}$, et al. Nipple-sparing mastectomy: can we predict the factors predisposing to necrosis? Eur J Surg Oncol. 2012;38:125-9.

13. Jensen JA, Orringer JS, Giuliano AE. Nipple-sparing mastectomy in 99 patients with a mean follow-up of 5 years. Ann Surg Oncol. 2011;18:1665-70.

14. Lago V, Maisto V, Gimenez-Climent J, Vila J, Vazquez C, Estevan R. Nipple-sparing mastectomy as treatment for patients with ductal carcinoma in situ: a 10-year follow-up study. Breast J. 2018;24:298-303. 